\title{
ABSTRACTS OF THE DUTCH SOCIETY OF CLINICAL PHARMACOLOGY AND BIOPHARMACY MEETING OF 7 OCTOBER 2008
}

\begin{abstract}
Polymorphisms of dopamine $D_{3}$, serotonin $2_{A}$ and 2 c receptors and orofaciolingual and limb-truncal dyskinesias in Russian psychiatric inpatients from Siberia

A. F. Y. Al Hadithy, ${ }_{1}^{1-4}$ S. A. Ivanova, ${ }_{1}^{5}$ P. Pechlivanoglou, ${ }^{6}$ A. Semke, O. Fedorenko, ${ }^{5}$ E. Kornetova, ${ }^{5}$ L. Ryadovaya, ${ }^{5}$ J. R. B. J. Brouwers, ${ }^{1,2}$ B. Wilffert, ${ }^{1,2}$ R. Bruggeman ${ }^{1,4}$ \& A. J. M. Loonen ${ }^{1,}$

'Pharmacotherapy and Pharmaceutical Care, University of Groningen, Groningen, The Netherlands (NL); ${ }^{2}$ Clinical Pharmacy and Pharmacology, Zorggroep Noorderbreedte and De Tjongerschans, Leeuwarden, NL; ${ }^{3}$ Department of Clinical Pharmacology, University Medical Center Groningen, Groningen, NL; ${ }^{4}$ Department of Psychiatry, University Medical Center Groningen, Groningen, NL; ${ }^{5}$ Mental Health Research Institute, Tomsk, Russian Federation; ${ }^{6}$ Groningen Research Institute of Pharmacy (GRIP), University of Groningen, Groningen, NL; ${ }^{7} G G Z$ Westelijk Noord-Brabant, Bergen op Zoom, The Netherlands
\end{abstract}

Introduction: Genetics of tardive dyskinesia has been studied in various ethnic groups, but not in Russians.

Aims: To study the association between orofaciolingual (TDof) and limb-truncal dyskinesias (TDIt) and polymorphisms of dopamine $D_{3}$, serotonin $2_{A}$, and 2 c receptors (Ser9Gly, $-1438 G>A$, and Cys23Ser, respectively) in Russian inpatients from Tomsk, Siberia.
Methods: Totally, 146 subjects were genotyped and rated for the presence of TDof and TDIt with AIMS items 1-4 and 5-7, respectively. Two-part model analyses, logistic and multivariate parametric regression were applied to assess the effects of different variables (e.g., allele-carriership, age, gender, and the use of antipsychotic and anticholinergic medication). About $95 \%$ of the subjects had clinically-established schizophrenia.

Results: In contrast to TDof, TDIt exhibited an association with Ser9Gly and Cys23Ser polymorphisms (9Gly-allele being predisposing for and the $23 \mathrm{Ser}$-allele being protecting against higher TDlt-scores). However, none of the polymorphisms studied could predict clinically important TDof or TDlt.

Conclusions: This is the first report on TD-pharmacogenetics in Russians. Although type I or II errors can not excluded, the present study suggests that the pharmacogenetic effects of Ser9Gly, $-1438 \mathrm{G}>\mathrm{A}$, and Cys23Ser polymorphism on TD may be different in Russians from those in other ethnic groups.

Our data suggest that none of the polymorphisms studied predict clinically present TDof or TDIt. However, Ser9Gly and Cys23Ser polymorphisms may affect the phenotypic variability of TDIt after its occurrence, but not that of TDof.

Future studies in larger samples of comparable ethnicity are warranted to support our findings.

\section{High intra-individual variability in pharmacokinetics of immunosuppressive drugs is a risk factor for poor long-term outcome after kidney transplantation \\ L. C. P. Borra, ' J. I. Roodnat, ${ }^{2}$ J. A. Kal, ${ }^{2}$ R. A. A. Mathot, ${ }^{1}$ W. Weimar ${ }^{2}$ \& T van Gelder ${ }^{1,2}$ \\ 'Dept. of Hospital Pharmacy, ${ }^{2}$ Dept. of Internal Medicine Erasmus MC, Rotterdam, The Netherlands}

Introduction: Inter-patient variability in the PK of immunosuppressive drugs and its determinants has been extensively studied. The importance of intra-patient variability in PK of immunosuppressive drugs for long-term outcome after kidney transplantation is still unclear.

Hypothesis: We hypothesized that high intra-individual variability in PK of the immunosuppressive drugs Tacrolimus and MMF would put patients at risk for periods of over- or under immunosuppression, and would thus lead to long-term chronic allograft nephropathy and graft loss after transplantation.

Methods: 297 patients transplanted between 1-1-2000 and 31-122004 were included in the study. The intra-individual variability in tacrolimus whole blood concentrations and mycophenolic acid plasma concentrations between 6-12 months post Tx was calculated. The patients were divided in low and high intra-individual variability groups, using the median variability as cut-off value. The mean variability was $14.9 \%$ and $28.8 \%$ for tacrolimus and MMF respectively. A COX regression analysis was performed using graft survival as time indicator. As primary outcome a composite endpoint consisting of graft loss, biopsy proven chronic allograft nephropathy and 'doubling in plasma creatinine concentration in the period between $t=12$ months post $T x$ and last follow-up' was used. Included covariates besides intra-individual variability of tacrolimus and MMF were sex, age, 'episode of biopsy proven acute rejection in the $1^{\text {st }}$ year post Tx','most recent $\%$ PRA pre Tx', 'highest \% PRA', 'nr. of previous transplantations', living donor, pre-emptive transplantation, HLA mismatch and delayed graft function post Tx.

Results: In the study population of 297 patients, 34 patients reached the primary endpoint of graft failure. Intra-individual variability of tacrolimus and 3 other covariates are significant risk factors for reaching the composite endpoint of failure (intraindividual tacrolimus variability $=0.006, \mathrm{BPAR}=0.007, \%$ PRA recent $=0.015, \%$ PRA $\max =0.032$ ). Intra-individual variability of MMF was not related to the composite endpoint of failure. (0.519) 


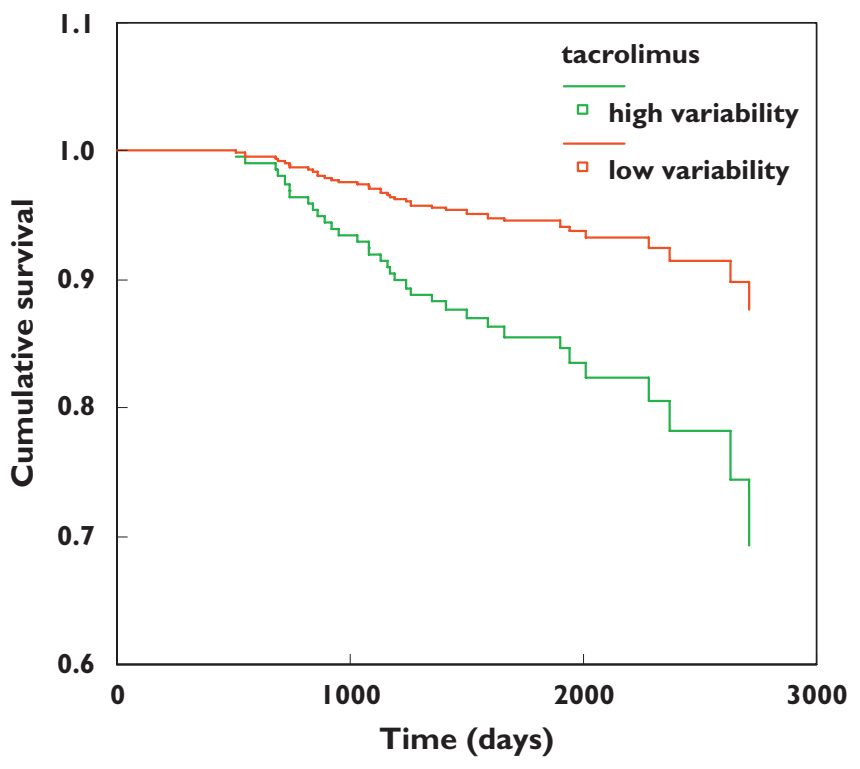

Conclusions: This study shows a significant relationship between high intra-individual variability in whole blood concentrations of tacrolimus and reaching the composite endpoint of graft failure. The results of this study did not show any relation between intraindividual variability of MMF and long-term graft failure. Possibly this difference is caused by a wider therapeutic window for MMF, compared to tacrolimus.

\section{Polypharmacy optimizing method (POM); effect on appropriate prescribing \\ A. C. Drenth-van Maanen, ${ }^{1}$ R. J. van Marum, ${ }^{1}$ W. Knol, ${ }^{1}$ C. M. J. van der Linden² \& P. A. F. Jansen' \\ 'Department of Geriatric Medicine, University Medical Centre, Utrecht, The Netherlands, ²Department of Geriatric Medicine, Catharina Hospital, Eindhoven, The Netherlands}

Objectives: Optimizing polypharmacy is often complex. Frequently critical appraisal of medication use leads to one or more changes. In order to assist general practitioners (GP's) to optimize polypharmacy, we have developed a method, based on 6 questions:

1) Is undertreatment present?

2) Does the patient adhere to the medication?

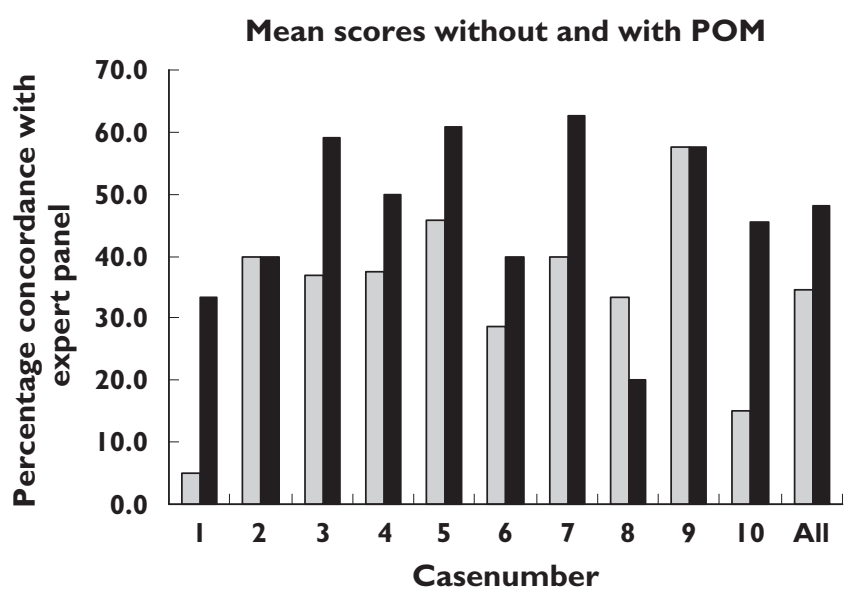

\section{Figure 1}

Concordance(\%) of appropriate decisions per case. Without ( $\square$ ); With (
3) Which drug(s) is (are) not indicated?

4) Which adverse effects are present?

5) Which clinically relevant interactions are to be expected?

6) Should the dosage, the dose frequency, and/or form of the drug be adjusted?

The aim of this study is to evaluate the usefulness of POM as a tool to improve appropriate prescribing of complex polypharmacy.

Methods: 45 GP's received out of 10 cases of geriatric patients, with a mean of $7.9 \pm 1.2$ problems treated with $8.7 \pm 3.1$ drugs, at random 2 cases. The first case was optimized without knowledge of POM. After a lecture about POM of one of us (RM) the second case was optimized with help of POM. All cases were conducted within 20 minutes. The outcomes were compared to appropriate answers, composed by consensus of an expert panel

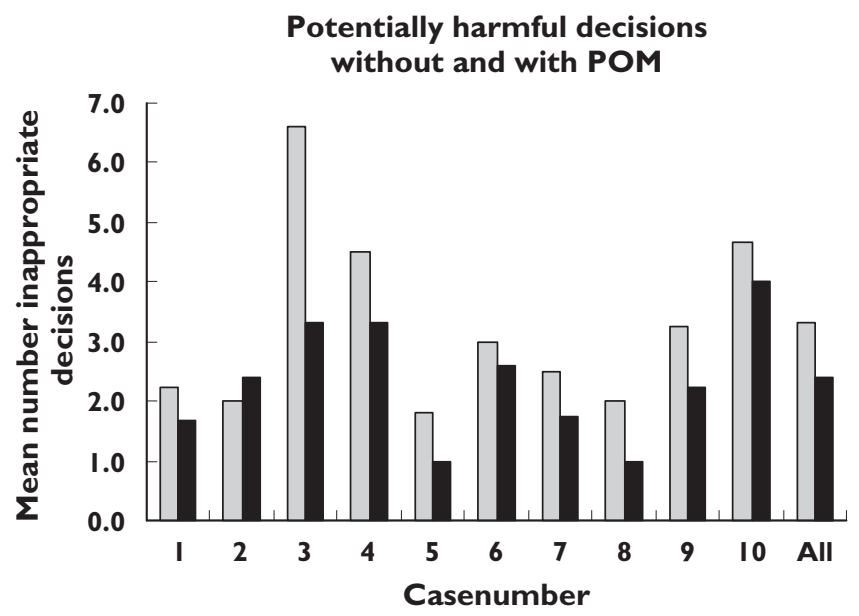

Figure 2

Number of potentially harmful decisions per case. Without ( $\square$ ); With 
of 4 geriatricians/clinical pharmacologists (RM, WK, CL, PJ). Data were analyzed, without knowing if POM was used or not, with a linear mixed effect model (AD).

Results: Use of POM showed a significant improvement of optimization. The percentage right decisions increased from $34.7 \%$ without POM to $48.1 \%$ with POM ( $p=0.0037$ ). The number of potentially harmful decisions decreased from 3.3 without POM to 2.4 with POM $(p=0.0046)$.

Conclusion: The Polypharmacy Optimizing Method improves appropriate prescribing of complex polypharmacy.

\section{The effect of seven genetic variants on the response to clopidogrel}

A. M. Harmsze, ${ }^{1}$ J. W. van Werkum, ${ }^{1}$ V. H. Deneer, ${ }^{1}$ H. J. Ruven,

O. H. Klungel, ${ }^{2}$ C. M. Hackeng, ${ }^{1}$ J. M. ten Berg' \& A. de Boer ${ }^{2}$

'St Antonius Hospital, Nieuwegein, The Netherlands and '2Utrecht

University for Pharmaceutical Sciences, Utrecht, The Netherlands

Introduction: Recently it was demonstrated that CYP2C19*2 in healthy subjects and patients with acute coronary syndrome was associated with impaired inhibition of ADP-induced platelet aggregation during clopidogrel treatment. Other genetic polymorphisms in the metabolism and pathway of action of clopidogrel may also contribute to the variable response.

Hypothesis: We hypothesized that the genetic variants of $A B C B 1$ (C1236T, G2677T/A, C3435T), CYP3A5 (*3), CYP2C19 $\left({ }^{*} 2,{ }^{*} 3\right)$ and P2Y1 (A1622G) have an effect on clopidogrel response in patients undergoing elective percutaneous coronary intervention ( $\mathrm{PCl}$ ) on dual antiplatelet therapy.

Methods: In a total of 428 patients undergoing elective $\mathrm{PCl}$ treated with clopidogrel and aspirin platelet function was evalu- ated by light transmittance aggregometry. Prior to $\mathrm{PCl}$ patients received a $300 \mathrm{mg}$ oral clopidogrel loading dose (1-5 days prior to $\mathrm{PCl}$, followed by $75 \mathrm{mg} /$ day) or used $75 \mathrm{mg} /$ day for $\geq 5$ days prior to PCl. Real-time PCR and RFLP were used for analysis of polymorphisms. Linear and logistic regression analysis was used to assess the associations between genetic variants and platelet function.

Results: In both treatment groups carriers of the CYP2C19*2 allele had significantly higher ADP-induced platelet aggregation values after 2, 5, 10 and $20 \mu \mathrm{M}$ ADP (all $p<0.01$ ). Associations remained significant after adjusting for potential confounders. After covariate adjustment, CYP2C19*2 was also associated with clopidogrel nonresponsiveness (defined as $20 \mu \mathrm{M}$ ADP-induced platelet aggregation >70\%); 75 mg-group: OR $3.6395 \% \mathrm{Cl}, 1.93-$ 6.82; 300 mg-group: OR $4.5695 \% \mathrm{Cl}$, 1.79-11.64. The ABCB1, CYP3A5 and P2Y1 genetic variants were not associated with clopidogrel response.

Conclusions: This study demonstrates that CYP2C19*2 is associated with blunted response to clopidogrel in patients undergoing elective $\mathrm{PCl}$ on dual antiplatelet therapy.

\section{The effect of proton pump inhibitors on the antiplatelet properties of clopidogrel is dependent on clopidogrel dosing regimen}

A. M. Harmsze, ${ }^{1,2}$ J. W. van Werkum, ${ }^{1}$ C. M. Hackeng, ${ }^{1}$ J. M. ten Berg, $^{1}$ H. J. Ruven, ${ }^{1}$ O. H. Klungel, ${ }^{2}$ A. de Boer ${ }^{2}$ \& V. H. Deneer ${ }^{1}$

'St Antonius Hospital, Nieuwegein, The Netherlands and 'Utrecht University for Pharmaceutical Sciences, Utrecht, The Netherlands

Introduction: Clopidogrel is a prodrug that requires a cytochrome (CYP)2C19-dependent conversion to become active. Concomitant use of drugs that are metabolized by the same enzyme, such as proton pump inhibitors (PPIs), might attenuate clopidogrel's platelet inhibitory effect.

Hypothesis: We hypothesized that concomitant use of PPIs attenuates the antiplatelet properties of clopidogrel in patients undergoing elective percutaneous coronary intervention ( $\mathrm{PCl}$ ).

Methods: Optical aggregometry (ADP) was performed in 428 patients on clopidogrel and aspirin. Almost one third of the patients (131) received a $300 \mathrm{mg}$ clopidogrel loading dose prior to $\mathrm{PCl}$ (mean 2.5 days), followed by $75 \mathrm{mg} /$ day. The remaining 297 patients were on chronic clopidogrel maintenance therapy of $75 \mathrm{mg} /$ day for more than 5 days prior to $\mathrm{PCl}$. Comparisons were performed on the basis of PPI use. Of all patients CYP2C19 genotypes were determined.

Results: Of all patients $22.9 \%$ was treated with PPIs. In the $300 \mathrm{mg}$ loading dose group, the magnitude of on-clopidogrel platelet reactivity was significantly higher in PPI users as compared to PPI non-users. This association remained significant after correction for potential confounders. In contrast, no significant differences in on-clopidogrel platelet reactivity were observed between PPI users and non-users in the group of patients on chronic maintenance therapy. The use of PPIs was associated with poor-responder status (defined as $20 \mu \mathrm{M}$ ADP-induced platelet aggregation $>70 \%$ ) in the $300 \mathrm{mg}$-group (OR 2.81, 95\% $\mathrm{Cl} 1.11-7.14)$ but not in the group on chronic maintenance therapy.

Conclusions: The efficacy of clopidogrel is attenuated by PPI use when administered as a loading dose. Chronic clopidogrel maintenance therapy, however, is not affected by concomitant PPI use. 


\author{
Prescribing in frail elderly; criteria for \\ drug selection \\ M. Huisman-Baron, ${ }^{1}$ L. van der Veen, ${ }^{2}$ E. N. van Roon, ${ }^{3}$ J. R. B. J. \\ Brouwers, ${ }^{3}$ P. A. F. Jansen' \& R. J. van Marum \\ 'Department of Geriatrics; University Medical Center; Utrecht, \\ ${ }^{2}$ Tjongerschans Hospital, Heerenveen, Heerenveen, ${ }^{3}$ Department of \\ Pharmacology, University of Groningen, Groningen, The Netherlands
}

Introduction: Elderly patients with multiple diagnoses and polypharmacy are at an increased risk of adverse drug events. Even within a single class of drugs (e.g. ACE-inhibitors, antipsychotics, beta-blockers) much variation in drug characteristics can exist. In this study, we investigated which criteria are relevant for selecting the right drug for frail elderly in clinical practice.

Methods: Based on a literature search in Pubmed and Embase, a list of 31 criteria used for evaluation of prescribing quality was constructed by the investigators. This list was presented to 51 experts ( 25 physicians, 26 pharmacists) who were asked to score each item for relevance in clinical practice on a scale from 1 to 10 (10 = highest relevance). The $75 \%$ items with the highest score were automatically included. Criteria with lowest scores (25\%) were debated by the authors.

Results: This strategy resulted in a set of 22 criteria, divided into four categories: effectiveness, safety, experience and convenience (table).

Conclusion: This criteria list reflects literature and clinical expert opinion and can be used for determination of appropriateness of a single drug for use in frail elderly compared to alternative drugs for the same indication or within the same class.
Table

\begin{tabular}{|c|c|}
\hline Criteria & $\begin{array}{l}\text { Scored } \\
\text { relevance }\end{array}$ \\
\hline \multicolumn{2}{|l|}{ I. Effectiveness } \\
\hline What is the evidence for effectiveness for this indication? & 8.11 \\
\hline What is the time until benefit? & 7.89 \\
\hline What is the number needed to treat? & 7.37 \\
\hline \multicolumn{2}{|l|}{ II. Safety } \\
\hline Which important drug-drug interactions can be expected? & 8.11 \\
\hline What are the most relevant side effects? & 8.07 \\
\hline $\begin{array}{l}\text { Are there important drug-disease interactions? } \\
\text { Pharmacokinetic aspects }\end{array}$ & 7.85 \\
\hline Is there a small therapeutic window? & 8.33 \\
\hline Is elimination of the drug depending on renal function? & 8.22 \\
\hline \multicolumn{2}{|l|}{ Pharmacodynamic aspects } \\
\hline Has the drug anticholinergic effects? & 8.41 \\
\hline Has the drug sedative effects? & 8.30 \\
\hline Has the drug orthostatic effects? & 8.28 \\
\hline Does the drug influence cognition? & 8.22 \\
\hline Does the drug influence the locomotor system? & 8.07 \\
\hline Does the drug influence haemostasis? & 8.07 \\
\hline Does the drug influence food intake? & * \\
\hline Has the drug cardiovascular side effects? & * \\
\hline \multicolumn{2}{|l|}{ III. Experience } \\
\hline Which advice is given in guidelines? & 8.50 \\
\hline What is the experience with the drug? & 8.09 \\
\hline Is the drug registered for the chosen indication? & 7.25 \\
\hline \multicolumn{2}{|l|}{ IV. Convenience } \\
\hline Dosage forms and packaging & 7.61 \\
\hline Dose frequency & 7.17 \\
\hline
\end{tabular}

*Not in questionnaire; additional criteria suggested by experts.

\section{Non-steroidal anti-inflammatory drugs (NSAID) and gastro-protection: development and validation of a clinical rule}

M. ten Katen, ${ }^{1}$ M. L. Loeffen, ${ }^{1}$ A. M. J. W. Scheepers-Hoeks, ${ }^{1}$ H. M. M. Korsten $^{2}$ \& R. J. E. Grouls ${ }^{1}$

'Dept. of Clinical Pharmacy and ${ }^{2}$ Anaesthesiology, Catharina Hospital, Eindhoven, The Netherlands

Introduction: Gastrointestinal complications are a common side effect of NSAIDs. Certain factors can increase the risk on this complication. A regional expert-group established a regional guideline 'Use of NSAIDs and gastro-protection'. This guideline defines a patient as high-risk in the presence of one severe or two or more cumulative risk factors. It is recommended to initiate prophylactic gastro-protection in these patients by a proton pump inhibitor. A Clinical Decision Support System (CDSS) can be used to develop a clinical rule to select these patients and provides the opportunity to generate alerts in case the treatment does not follow the guideline.

Guideline: Severe risk factors: age over 70 years, history of (complications of) an ulcus, untreated H. Pylori-infection.
Cumulative risk factors: Age 60 to 70 years, use of anticoagulants, use of corticosteroids, use of Selective Serotonin Reuptake Inhibitors (SSRIs), debilitating rheumatoid arthritis, heartfailure, diabetes, high-dose NSAID treatment.

Aim: To develop and validate a clinical rule to guide gastroprotection during the use of NSAIDs, using the CDSS Gaston (Medecs), and to analyse pro- and retrospectively the compliance to the guideline and the potential impact of implementation of this clinical rule in the Catharina Hospital, Eindhoven (CZE).

Methods: The clinical rule was developed following a PlanDo-Check-Act cycle. The rule was retrospectively tested on 3047 patients with an admission day between the $1^{\text {st }}$ of april and the $31^{\text {st }}$ of may 2008 at the CZE. This test selects the patients that use a NSAID for more than one day. The prospective test includes 694 patients admitted to the CZE between the $12^{\text {th }}$ and the $18^{\text {th }}$ of july 2008. A signal was generated for each day of a high-risk patient using a NSAID and not using a gastro-protective drug.

Results: The retrospective test reveals that 535 patients used a NSAID more than one day. 225 of these patients (42.1\%) generated one or more signals. The prospective test selected 101 patients that used an NSAID for one or more days, among them 
40 that lacked gastro-protection although recommended by the guideline. In our hospital setting this is an average of 5 to 6 patients per day.

Conclusion: On the basis of the retrospective and prospective results it can be concluded that approximately $40 \%$ of the users of NSAIDs were not treated according to the guideline which can lead to severe gastrointestinal complications. The clinical rule for gastro-protection selects these patients, generates a message and draws a physician's attention to compliance to the guideline. In this way, implementation of this clinical rule can improve medication safety.

\section{Therapeutic drug monitoring and aminoglycosides: development and validation of a clinical rule}

M. ten Katen, ${ }^{1}$ M. L. Loeffen, ${ }^{1}$ A. M. J. W. Scheepers-Hoeks, ${ }^{1}$ A. N.

Roos, ${ }^{2}$ H. M. M. Korsten ${ }^{3}$ \& R. J. E. Grouls ${ }^{1}$

'Dept. of Clinical Pharmacy, ${ }^{2}$ Intensive Care, ${ }^{3}$ Anaesthesiology,

Catharina Hospital, Eindhoven, The Netherlands

Introduction: The efficacy and toxicity of aminoglycosides is concentration-dependent. The activity is increased by higher peak concentrations and the toxicity by higher trough concentration. Interindividual kinetic differences hinder the formulation of a standard dosage and it is therefore recommended to adjust the dose based on therapeutic drug monitoring (TDM). In the Catharina Hospital Eindhoven a protocol guides the process of 'TDM and aminoglycosides'.

A Clinical Decision Support System (CDSS) can be used to develop a clinical rule in order to select the users of aminoglycosides that are not monitored according to this guideline. A CDSS provides the opportunity to generate alerts for these patients.

Guideline: The guideline is formulated in a clinical rule based on an expert opinion. TDM needs to be applied:

- within 48 hours after an initial prescription.

- within 48 hours after a dose adjustment.

- within 4 days after the last aminoglycoside measurement.

- within 48 hours after a change in renal function of more than $20 \%$.
Aim: To develop and validate a clinical rule to guide TDM of aminoglycosides, using the CDSS Gaston (Medecs), and to analyse retrospectively the compliance to the guideline 'TDM and aminoglycosides' and the potential impact of implementation of this clinical rule.

Methods: The clinical rule was developed following a PlanDo-Check-Act cycle. The rule was retrospectively tested on 3047 patients with an admission day between the $1^{\text {st }}$ of april and the $31^{\text {st }}$ of may 2008 at the CZE. This test selects the patients using an aminoglycoside for more than 3 days and generates a message if the patient is not monitored in time according to the guideline.

Results: The retrospective test shows that 55 patients used an aminoglycoside for more than 3 days. 10 patients were not monitored in time after an initial prescription and 22 patients showed an interval of more than 3 days with the last aminoglycoside measurement. In all, 23 different patients (42\%) generated one or more messages.

Conclusion: Based on the retrospective results it can be concluded that $42 \%$ of the users of aminoglycosides are not monitored according to the hospital guideline during one or more days. Twenty-three patients generated 32 different alerts in two months. Based on this number it is estimated that this clinical rules leads to 3 to 4 messages per week after implementation in the Catharina Hospital and can substantially improve the process of TDM.

\author{
Analysis of regional differences in prescription \\ rates of trastuzumab (Herceptin ${ }^{\circledR}$ ) for breast \\ cancer in the Netherlands \\ G. Klappe-Sabadi, ${ }^{1}$ F. G. A. Jansman, ${ }^{2}$ H. A. Honkoop, ${ }^{3}$ R. Otter, \\ P. H. B. Willemse ${ }^{5}$ \& J. R. B. J. Brouwers ${ }^{6}$ \\ 'Department of Clinical Pharmacy, Isala Klinieken, Zwolle; \\ ${ }^{2}$ Department of Clinical Pharmacy, Deventer Ziekenhuis, Deventer; \\ ${ }^{3}$ Department of Medical Oncology, Isala Klinieken, Zwolle, \\ ${ }^{4}$ Comprehensive Cancer Center North-East, Groningen; ${ }^{5}$ Department \\ of Medical Oncology, University Medical Center Groningen, Groningen; \\ ${ }^{6}$ Groningen University Institute for Drug Exploration (GUIDE), \\ Groningen, The Netherlands
}

Introduction: In 2005, the Dutch Breast Cancer Association (BVN) investigated the prescription rates by oncologists of trastuzumab (Herceptin ${ }^{\circledR}$ ) in different regions of the Netherlands in 2004. Their survey demonstrated that the highest use of trastuzumab was in the south-west and the lowest use in the mid-north of the Netherlands. The BVN speculated that oncologists in the mid-north did not prescribe trastuzumab according to the (national) guidelines and that this innovative drug was withheld to patients because of high costs. The aim of our study was to analyze the factors that influenced the prescription rates of oncologists in regions with lowest versus highest use of trastuzumab in the Netherlands.

Methods: Oncologists, pathologists and hospital pharmacists working in the south-west (highest use: region A) and the midnorth (lowest use: region B) of the Netherlands were interviewed. Data were retrieved from January 2000 until December 2005. The following parameters were determined: number of HER2/neu positive patients, number of patients treated with trastuzumab, number of patients with an indication for trastuzumab, amount of clinical trials being performed with trastuzumab, age distribution, treatment regimens, reimbursement of costs, methods to determine HER2/neu status, incidence of breast cancer, obstructions for use of trastuzumab, guidelines for treatment of breast cancer, presence of a multidisciplinary treatment team, co morbidity, and number of patients refusing trastuzumab or with a contra-indication for use. 
Results: In $50 \%$ of the hospitals in regions $\mathrm{A}$ and $\mathrm{B}$, there were no obstructions for the use of trastuzumab; no difference was found between regions ( $p>0.05$ ). However, trastuzumab was used less frequently in region B (vials/1000 inhabitants). This lower use was associated with more restricted guidelines in region $B(p<0.001)$, limitations concerning diagnosis of the HER2/neu status ( $p<0.001)$, and a lower percentage of HER2/ neu positive patients $(p=0.027)$. Since $2004,70 \%$ of the women with metastatic breast cancer in region $A$ received trastuzumab concomitant with regular chemotherapy as a first line treatment. Trastuzumab was predominantly used as a second line treatment in region $B$. In this region, the shift towards first line treatment was made in 2005.

Conclusions: In both regions, trastuzumab was used according to the Dutch guidelines as a second line treatment for metastatic breast cancer. However, in the region with the highest use (southwest), trastuzumab was initiated ahead of treatment guidelines for HER2/neu positive metastatic breast cancer. Moreover, in the mid-north of the Netherlands, the percentage of HER2/neu over expression (positive) in the population of breast cancer patients was lower compared to the south-west region.

\section{Prevention of opioid induced constipation using a clinical rule}

M. L. Loeffen, ${ }^{1}$ M. ten Katen, ${ }^{1}$ A. M. J. W. Scheepers-Hoeks, ${ }^{1}$ J. A. van

Suijlekom, ${ }^{2}$ H. M. M. Korsten² \& R. J. E. Grouls'

'Department of Clinical Pharmacy and ${ }^{2}$ Department of

Anesthesiology, Catharina-hospital Eindhoven, The Netherlands

Background: Because of the high prevalence of opioid induced constipation (OIC), the prophylactic use of a laxative is widely recommended. However, not all patients get a laxative prescribed when opioid treatment is initiated. Third-generation patient based medication safety systems, also called computerized decision support systems (CDSS), can identify patients who lack prophylactic treatment. A CDSS uses clinical rules to generate patient based recommendations.

Aim: The aim of this research is to develop and validate a clinical rule that identifies patients who have an opioid but no laxative prescribed and to determine the potential impact of this rule.

Methods: A clinical rule that identifies patients who lack a prophylactic laxative to prevent OIC is developed using the CDSS Gaston (Medecs). The Plan-Do-Check-Act cycle is followed to create a reliable rule. The content is both based on literature and expert opinion. To determinate the potential impact, the clinical rule is tested retrospectively on all patients admitted in January and March 2008 and prospectively for 15 days in July and August
2008 on the patient database of the Catharina-hospital Eindhoven, the Netherlands (CZE).

For prospective testing, the clinical rule is designed to classify recommendations as high priority or normal priority, depending on the risk of the development of OIC.

High priority messages should be delivered directly to the clinician, while normal priority messages should be delivered to the nurses.

Reliability is measured by manual validation of the recommendations and expressed as the positive predictive value (PPV). The potential impact is measured by counting the number of recommendations that the CDSS generates.

Results: A total of 3313 patients were admitted in January and March 2008. Of these patients, 791 had an opioid prescribed. 536 (67.8\%) of them did not use a prophylactic laxative during one or more days. During prospective testing, a total of 656 recommendations to prescribe a prophylactic laxative are generated, of which 80 are classified as high priority. The most frequently generated high priority recommendations involve patients who have been prescribed morphine $(n=31)$, fentanyl $(n=27)$ or oxycodon $(n=10) .99$ of 100 validated messages are correct. This results in a PPV of $99 \%$.

Conclusion: A high percentage of opioid-using patients do not get a prophylactic laxative prescribed. This research has shown that a reliable clinical rule can be developed to identify patients who need the prescription a prophylactic laxative. When this clinical rule is implemented and used in daily practice it will probably reduce the prevalence of OIC.

\section{Drug clearance during CVVH in intensive care patients. An algorithm to assist initial dosing by predicting the sieving coefficient \\ C. M. Samson-Corbeij, ${ }^{1}$ R. J. E. Grouls, ${ }^{1}$ A. N. Roos, ${ }^{2}$ K. Koppenol, ${ }^{2}$ \\ P. Douwes-Draaijer ${ }^{3}$ \& E. W. Ackerman ${ }^{1}$ \\ 'Departments of Clinical Pharmacy, ${ }^{2}$ Critical Care Medicine, 3 Internal \\ Medicine and renal diseases, Catharina-hospital, Eindhoven, The \\ Netherlands}

Introduction: CVVH is widely used in hemodynamic instable patients with acute renal failure. Quantative information on the clearance and kinetics of drugs during CVVH is limited to a few antibiotics. To assist in initial dosing we developed an algorithm based on ex vivo experiments using calf blood to predict the sieving coefficient. The sieving coefficient (SC) is represented by proteinbinding (\%PB), partition coefficient octanol/ water $(\log \mathrm{P})$, charge $(\mathrm{C})$ and molecular weight $(\mathrm{Mw})$ : $\mathrm{SC}_{\text {predicted ex vivo }}=0,826-$ $0,006 \%(\mathrm{~PB})-0,055(\log \mathrm{P})-0,085(\mathrm{C})+0,000095(\mathrm{Mw})$. (SamsonCorbeij et al. 2006, 2007)

Aim: In the present study we validated the ex-vivo model in CVVH-patients admitted to the Intensive Care Unit of the Catharina-hospital in Eindhoven.

Methods: Samples were collected during CVVH with a PRISMA CFM hemofiltration machine, AN69 HF 0,9 $\mathrm{m}^{2}$, blood flow rate $150 \mathrm{ml} / \mathrm{min}$, ultrafiltration rate $1500 \mathrm{ml} / \mathrm{h}$, centrifuged and frozen at $-20^{\circ} \mathrm{C}$ until analysis. All analyses were performed by validated methods. By determining drug concentrations in de bloodline 
pre filter, post filter and in the ultrafiltrate, we calculated the SC and compared these with the $\mathrm{SC}_{\text {predicted ex vivo, }}$ by regression analysis and assessment for predictive performance (RMSE $=1 / \mathrm{n}^{i} \Sigma \mathrm{pe}_{\mathrm{i}}{ }^{2}$, $M E=1 / n^{i} \Sigma p_{i}($ Goerdt et al, 1997). The study was approved by the authorised ethics committee of the Catharina-hospital.

Results: Figure 1 shows a positive correlation between SC predicted with the ex-vivo formula and SC observed in vivo.

Conclusions: For initial dosing the sieving coefficient in vivo for drugs dispensed during CVVH with a AN69 membrane in critically ill patients, admitted to the ICU, can be estimated according the equation: $\mathrm{SC}_{\text {predicted in vivo }}=0,966-0,007 \%(\mathrm{~PB})-0,064(\log \mathrm{P})-$ $\mathbf{0 , 0 9 9}(\mathrm{C})+\mathbf{0 , 0 0 0 1 1}(\mathrm{Mw})+\mathbf{0 , 0 1 7 1}\left(R^{2} 0,83\right)$. Because of intraindividual variation and possible interindividual variation, therapeutic drug monitoring remains required to refine the dosing regime during pharmacotherapy.
Goerdt et al., British Journal of Clinical Pharmacology, 1997; 44: 261-265.

Samson-Corbeij et al., British Journal of Clinical Pharmacology, 2007; 63(4): 506-507.

\section{SC predicted ex vivo versus SC determined in vivo}

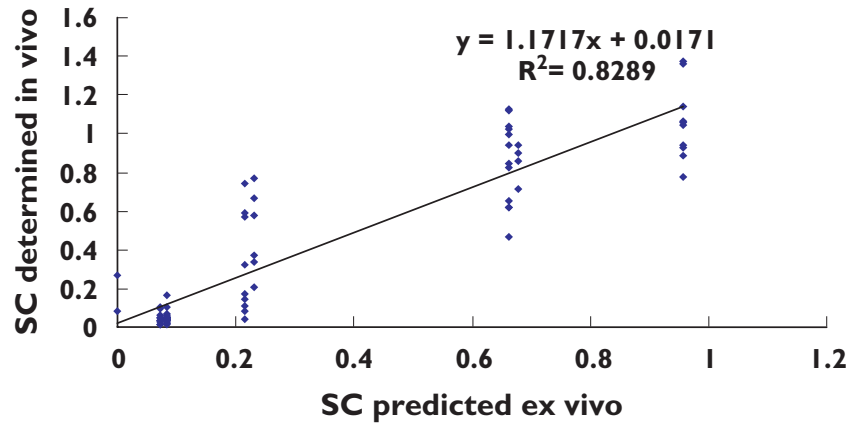

Results: From 80 patients (age 19-76 years), a full $A_{U} C_{M P A}$ and $A U C_{\text {act }}$ could be calculated on day 6 (range 3-11) posttransplantation. In 12 patients the IMPDH2 3757C allele was found ( 10 heterozygous and 2 homozygous individuals), giving an allele frequency of $6.9 \%$. None of the other SNPs were found in these cohort.

\& T. van Gelder

'Erasmus University Medical Center, Rotterdam, The Netherlands; ${ }^{2}$ Charité University, Berlin, Germany; ${ }^{3}$ F Hoffmann-La Roche, Basel, Switzerland

Introduction: Mycophenolic acid (MPA), the active metabolite of MMF, reversibly inhibits inosine monophosphate dehydrogenase (IMPDH) to prevent graft rejection. It is thought that pretransplantation, total IMPDH activity mostly consists of constitutively expressed IMPDH type 1, whereas post-transplantation IMPDH type 2 from activated lymphocytes has the major contribution. The considerable post-transplantation inter-individual variability in IMPDH activity may be caused by functional polymorphisms in the IMPDH2 gene.

Aim: This paper compares IMPDH activity with functional polymorphisms in the IMPDH2 gene.

Methods: A cohort of 100 patients was prospectively monitored for pharmacokinetics (MPA plasma levels) and pharmacodynamics (IMPDH activity) of MMF treatment. Patients received MMF, tacrolimus and steroids for immunosuppressive therapy. Blood samples were taken pre- and post-transplantation (pre-morning dose, $0.5,1,2,6$ and $12 \mathrm{~h}$ after MMF intake). The enzyme activity of IMPDH in PBMCs was measured using a validated HPLC method. IMPDH activity and MPA exposure was calculated as the area under the activity or concentration-time curve (AUC act and $A U C_{M P A}$ ) using a linear trapezoidal rule. Genomic DNA from all patients was analyzed for 8 IMPDH2 SNPs.

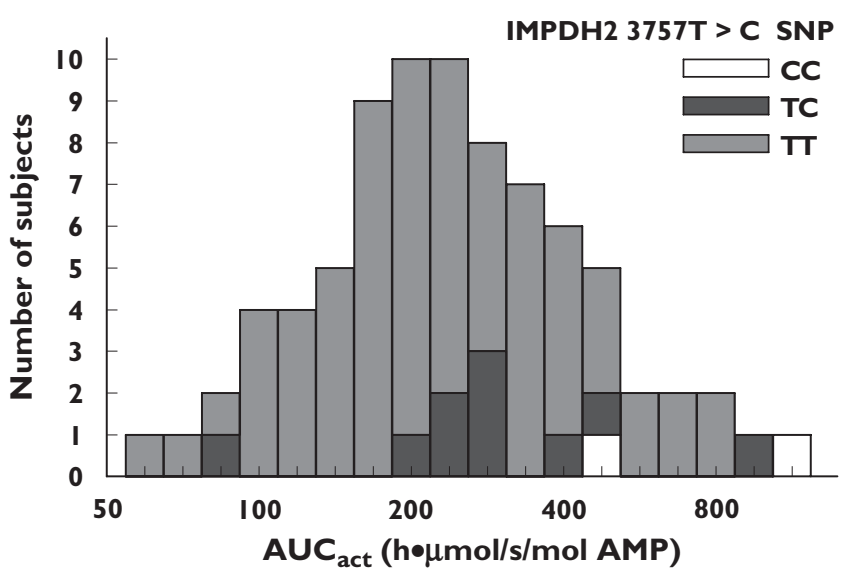

The presence of an IMPDH2 3757C allele correlated with a significantly higher total IMPDH activity in post transplantation patients under MMF exposure (AUC act $420 \pm 320$ vs. $265 \pm$ $160 \mathrm{~h}^{*} \mu \mathrm{mol} / \mathrm{s} / \mathrm{mol}$ AMP; $\left.\mathrm{p}=0.035\right)$, whereas the polymorphism was not correlated with pre-transplant IMPDH activity (54.4 \pm 44.2 vs. $53.3 \pm 37.2 h^{*} \mu \mathrm{mol} / \mathrm{s} / \mathrm{mol} A M P ; p=0.82$ ). AUC MPA $_{\text {was not }}$ significantly different between the two IMPDH genotype groups $\left(42.0 \pm 18.0\right.$ and $\left.35.5 \pm 19.1 h^{*} \mathrm{mg} / \mathrm{L} ; \mathrm{p}=0.18\right)$.

Conclusion: The IMPDH2 3757T>C polymorphism is significant correlated with IMPDH activity, measured as $A U C_{\text {act }}$ after renal transplantation. The presence of one or two IMPDH2 3757 $\mathrm{C}$-alleles gives a higher $\mathrm{AUC}_{\text {act. }}$. Whether this genotype is also associated with increased risk of acute rejection will be subject of future studies. 


\author{
IMPDH activity on day 6 after kidney \\ transplantation is significantly related to the risk \\ of acute rejection in MMF treated patients \\ F. Sombogaard, ${ }^{1}$ R. A. A. Mathot, ${ }^{1}$ P. Glander, ${ }^{2}$ W. Weimar' \& \\ T. van Gelder ${ }^{1}$ \\ 'Erasmus University Medical Center, Rotterdam, The Netherlands; \\ ${ }^{2}$ Charité University, Berlin, Germany
}

Introduction: Mycophenolic acid (MPA) - the active metabolite of MMF - inhibits the enzyme inosine monophosphate dehydrogenase (IMPDH). Monitoring IMPDH activity, as a measure of the immunosuppressive effect of MMF, could be a target to optimize MMF therapy. A previous study reported that high pre-transplant IMPDH activity was associated with biopsy proven acute rejection (BPAR) of the graft. So far, data on post-transplant IMPDH activity and BPAR are not available. In this study we investigated the relationship between IMPDH activity and risk of BPAR in renal transplant patients.

Methods: A cohort of 100 patients, transplanted between 4/06 and 9/07, was prospectively monitored for pharmacokinetics (MPA plasma levels) and pharmacodynamics (IMPDH activity) of MMF treatment. Patients received triple immunosuppressive therapy, consisting of MMF, tacrolimus and steroids. Blood samples were taken pre transplantation and on day 6 post transplantation pre-morning dose and 0.5, 1, 2, 6 and $12 \mathrm{~h}$ after MMF intake. The enzyme activity of IMPDH in PBMCs was measured using a validated HPLC method. IMPDH activity and MPA exposure was calculated as the area under the activity or concentration-time curve $\left(A \cup C_{a c t}\right.$ and $\left.A U C_{M P A}\right)$ using a linear trapezoidal rule. All acute rejection episodes were biopsy proven (Banff classification grade IA or higher). This interim analysis includes patients with a follow-up after transplantation of at least two months.
Results: Samples for IMPDH activity measurement were available from 80 patients (age 19-76 years) on day 6 (range 3-11) post transplantation. In 17 (21\%) of the patients a BPAR was documented within one year after transplantation. The mean $A U C_{a c t}$ of patients with BPAR was significantly higher compared to nonBPAR patients $\left(394 \pm 287\right.$ and $255 \pm 156 \mathrm{~h}^{*} \mu \mathrm{mol} / \mathrm{s} / \mathrm{mol}$ AMP, $\mathrm{p}=$ $0.017)$. The IMPDH activity $0.5 \mathrm{~h}$ and $2 \mathrm{~h}$ after MMF intake was also significantly higher in patients with a BPAR $(0.5 \mathrm{~h}: 35 \pm 26 \mathrm{vs} .21 \pm$ $15 \mathrm{~h}^{*} \mu \mathrm{mol} / \mathrm{s} / \mathrm{mol}$ AMP; $\mathrm{p}=0.028 ; 2 \mathrm{~h}: 23 \pm 15 \mathrm{vs} .15 \pm 11 \mathrm{~h} * \mu \mathrm{mol} /$ $s / \mathrm{mol} A M P ; p=0.013)$. No differrences were found between pretransplant IMPDH activity of the two groups (48 \pm 27 vs. $54 \pm$ $41 \mathrm{~h}^{*} \mu \mathrm{mol} / \mathrm{s} / \mathrm{mol}$ AMP; $\left.\mathrm{p}=0.97\right)$. The mean $\mathrm{AUC}_{\mathrm{MPA}}$ of patients with BPAR was not significantly different from non-BPAR patients $\left(36.5 \pm 17\right.$ vs. $\left.37.0 \pm 20 h^{*} \mathrm{mg} / \mathrm{L} ; \mathrm{p}=0.95\right)$.

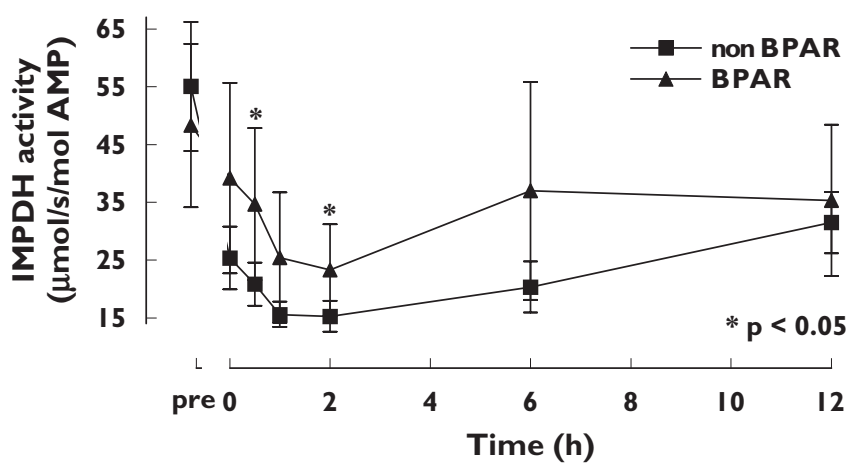

Conclusion: In the largest patient series to date, IMPDH activity, measured as $A U C_{\text {act }}$ on day 6 and $0.5 \mathrm{~h}$ and $2 \mathrm{~h}$ post-intake, is significantly correlated with BPAR after renal transplantation. Monitoring IMPDH activity may be a promising, novel biomarker to optimize MMF treatment in renal transplant patients.

\section{The influence of reduced activity of VKORC1 and CYP2C9 genotypes on anticoagulation in initial treatment with acenocoumarol \\ Teichert M, ${ }^{1,2}$ Stricker BHCh ${ }^{1,3,4}$ \& Visser LE $E^{1,5}$ \\ 'Department of Epidemiology \& Biostatistics, Erasmus Medical Center, Rotterdam, The Netherlands \\ ${ }^{2}$ Scientific Institute Dutch Pharmacists, The Hague, The Netherlands \\ ${ }^{3}$ Department of Internal Medicine, Erasmus Medical Center, \\ Rotterdam, The Netherlands \\ ${ }^{4}$ Drug Safety Unit, Inspectorate for Health Care, The Hague, The \\ Netherlands \\ ${ }^{5}$ Department of Hospital Pharmacy, Erasmus Medical Center, \\ Rotterdam, The Netherlands}

Introduction: We investigated polymorphisms in genes of Vitamin K Epoxide Reductase Complex subunit 1 (VKORC1) and Cytochrome P450C9 (CYP2C9) in relation to the international normalized ratio (INR) during the first 6 weeks of treatment with acenocoumarol and the effect on dosage in a population based cohort.

Methods: In 1525 patients from the Rotterdam Study on acenocoumarol the VKORC1 $1173 C>T$ and the CYP2C9*2 and *3 allele vari- ants were determined. Data on INR and coumarin dosage came from a regional anticoagulation clinic. After a standard starting dose of acenocoumarol, the first INR and risk of overanticoagulation were compared between genotypes. Furthermore the risk of severe overanticoagulation during initiation period and mean dosage at the end of the initiation period were analyzed.

Results: After an initial standard dosage of acenocoumarol, with the number of VKORC1 T alleles the risk on severe overanticoagulation increased 1,9 times $(95 \% \mathrm{Cl} 1,1-3.2)$ and all five bleeding events were in patients with one variant allele of either one of the genes. Acenocoumarol dosage at the end of the initiation period was decreased with 5,1 mg/week $(95 \% \mathrm{Cl}-5,5-(-4,7))$ by additional VKORC1 T-allele and further per CYP2C9 variant allele with $1,8 \mathrm{mg} /$ week $(95 \% \mathrm{Cl}-2,1-(-1,5))$.

Conclusion: After an initial standard dosing regime with acenocoumarol, patients with variant alleles of the VKORC1 and CYP2C9 genes were at increased risk of severe overanticoagulation. Personalized dosage of coumarins by genotyping prior to coumarin therapy might contribute to prevent serious side effects, and clinical utility of this policy should be confirmed in large prospective studies. 


\section{Development and testing of a protocol to aid decision making after acute intoxication}

M. Vroegop, ${ }^{1}$ F. van Summeren, ${ }^{2}$ R. Aarnoutse, ${ }^{3}$ G. J. van der Wilt,

F. G. A. Jansman, ${ }^{5}$ C. Hoedemaekers ${ }^{6} \&$ C. Kramers $^{2}$

Departments of 'Emergency Medicine, ${ }^{2}$ Pharmacology-Toxicology,

${ }^{3}$ Clinical Pharmacy, ${ }^{4}$ Medical Technology Assessment, ${ }^{6}$ Intensive Care,

University Center Nijmegen, PO Box 9101, 6500 HB, Nijmegen, The

Netherlands. ${ }^{5}$ Department of Clinical Pharmacy, Deventer Ziekenhuis

and ${ }^{6}$ Groningen University Institute for Drug Exploration (GUIDE),

Groningen, The Netherlands

Introduction: There are no guidelines if and where to admit patients after an acute intoxication. We aimed to develop and prospectively test a protocol to support admission decisions in patients with an acute intoxication. We compared this protocol with the advise given by the Dutch Poison and Information Centre.

Hypothesis: Usage of the protocol leads to a more efficient care for patients after an acute-intoxication.

Materials and Methods: A protocol was developed based on a previously conducted study of determinants of need for in-hospital treatment in patients after acute intoxication. This protocol was tested in a prospective cohort of 169 acute intoxications. In this cohort it was assessed which patients had eventually received a treatment that made admission necessary. Based on these patients sensitivity and specificity of the protocol for admission was determined. These were compared with the advice provided by the Dutch Poison and Information Centre.

Results: In the prospective study, 76 out of 169 acute intoxications patients were admitted (45\%). Of these, 25 patients received treatment ( 7 because of symptoms and 18 preventive treatments). All of the acute intoxications that received treatment were identified by the protocol (sensitivity 100\%). Out of the intoxications that did not receive treatment, in 95 patients no admission was advised by the protocol (specificity 66\%). The advice of the Dutch Poison and Information Centre had the same sensitivity, but a lower specificity (19-34\%).

Conclusion: This protocol can support the physician in decisionmaking in acute intoxications. It may lead to a more efficient admission policy.

\section{Limited sampling strategies for mycophenolic acid in patients with autoimmune disease \\ B. C. M. de Winter, ${ }^{1}$ I. Neumann, ${ }^{2}$ T. van Gelder, ${ }^{1}$ R. M. van Hest ${ }^{1}$ \& \\ R. A. A. Mathot ${ }^{1}$ \\ 'Department of Hospital Pharmacy, Erasmus University Medical \\ Center, Rotterdam, The Netherlands; 'Department of Nephrology, \\ Wilhelminenspital, Vienna, Austria}

Introduction: Mycophenolate mofetil (MMF) is increasingly used for the treatment of autoimmune diseases (AID). In renal transplant recipients it has been demonstrated that adjustment of MMF dose according to the area under the plasma concentration versus time curve (AUC) of mycophenolic acid (MPA), the active moiety of MMF, improves clinical outcome. ${ }^{[1]}$ The aim of this study was to develop a maximum a posteriori (MAP) Bayesian method to estimate MPA AUC $0-12$ in AID patients using only a limited number of samples. The predictive performance of the MAP Bayesian method was compared with that of a multilinear regression method.

Methods: Full MPA concentration vs time curves (13 samples per curve) were available from 38 patients with AID (26 with antineutrophil cytoplasmic antibody-associated vasculitis; 12 with systemic lupus erythematosus) treated with mycophenolate mofetil (MMF). Nonlinear mixed-effect modeling (NONMEM) was used to develop a population pharmacokinetic (PK) model. Patients were divided in an index- and a validation dataset. The population PK model derived from the index dataset was used to develop several Bayesian estimators. The Bayesian estimators were used to predict $A U C_{0-12}$ on basis of a limited number of blood samples. The bias and precision of these predictions were compared with those of limited sampling strategies developed with multilinear regression.

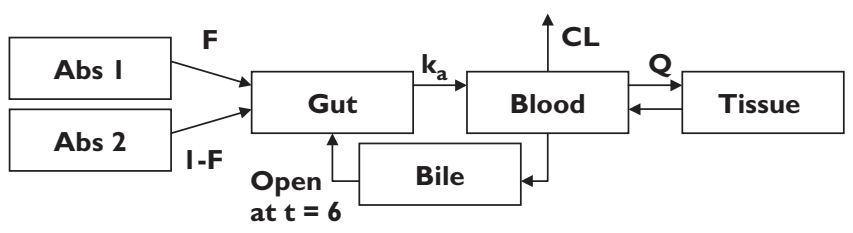

Figure

Population pharmacokinetic model of MPA

Results: A double absorption model, with first order absorption and elimination was used to describe the data (figure). The PK model accounted for the enterohepatic recirculation of MPA. Using 1 to 4 samples MPA AUC $_{0-12}$ was adequately estimated by the Bayesian method. Bias was not significantly different from zero and precision was lower than $27 \%$ (table). The predictive performance values of the multilinear regression method were comparable.

\section{Table}

Bias and precision of the limited sampling strategies

\begin{tabular}{|c|c|c|c|c|}
\hline \multirow[b]{2}{*}{ Samples } & \multicolumn{2}{|l|}{ Linear regression } & \multicolumn{2}{|c|}{ Bayesian estimator } \\
\hline & $\begin{array}{l}\text { Bias }(95 \% \mathrm{Cl}) \\
(\%)\end{array}$ & $\begin{array}{l}\text { Precision } \\
(\%)\end{array}$ & $\begin{array}{l}\operatorname{Bias}(95 \% \mathrm{Cl}) \\
(\%)\end{array}$ & $\begin{array}{l}\text { Precision } \\
(\%)\end{array}$ \\
\hline$n=1(t=0)$ & $3.4(-9.8-16.6)$ & 26.8 & $-5.3(-18.3-7.7)$ & 26.8 \\
\hline $\begin{array}{c}\mathrm{n}=2(\mathrm{t}=0,1 / \\
0,0.7)\end{array}$ & $4.8(-7.4-17.0)$ & 25.1 & $-5.6(-17.6-6.4)$ & 24.9 \\
\hline$n=3(t=0,1,3)$ & $0.8(-10.4-12.0)$ & 22.6 & $-5.5(-16.6-5.6)$ & 23 \\
\hline$n=4(t=0,1,3,6)$ & $-0.4(-9.0-8.1)$ & 17.3 & $-5.5(-14.4-3.5)$ & 18.9 \\
\hline
\end{tabular}


Conclusion: MAP Bayesian estimators were developed for the estimation of MPA AUC $_{0-12}$ in AID patient taking MMF. The predictive performance of the MAP Bayesian estimators was good and comparable to those of the multilinear regression method. Due to its flexibility with respect to sample times the MAP Bayesian method may be preferred over the multilinear regression method.

1 Le Meur, et al. Am J Transplant 2007; 7(11): 2496-503.

\author{
Population pharmacokinetics of mycophenolic acid: \\ comparison between enteric-coated mycophenolate \\ sodium and mycophenolate mofetil in renal \\ transplant patients \\ B. C. M. de Winter, ${ }^{1}$ T. van Gelder, ${ }^{1}$ P. Glander, ${ }^{2}$ D. Cattaneo, \\ H. Tedesco-Silva, ${ }^{4}$ I. Neumann, ${ }^{5}$ L. Hilbrands, ${ }^{6}$ R. M. van Hest, ${ }^{1}$ \\ M. D. Pescovitz, K. Budde ${ }^{7}$ \& R. A. A. Mathot ${ }^{1}$ \\ 'Erasmus University Medical Center, Rotterdam, The Netherlands; \\ ${ }^{2}$ Charité-Universitätsmedizin Berlin, Germany; ${ }^{3}$ Mario Negri Institute for \\ Pharmacological Research, Bergamo, Italy; ${ }^{4}$ Hospital do Rim e \\ Hipertensao, Universidade Federal de Sao Paulo, Brazil; \\ ${ }^{5}$ Wilhelminenspital, Vienna, Austria; ${ }^{6}$ Radboud University Nijmegen \\ Medical Center, Nijmegen, The Netherlands and 'Indiana University \\ Medical Center, Indianapolis, Indiana, USA
}

Aim: The pharmacokinetics of mycophenolic acid (MPA) were compared between renal transplant patients receiving either mycophenolate mofetil (MMF) or enteric-coated mycophenolate sodium (EC-MPS).

Methods: MPA concentration versus time profiles were included from EC-MPS- $(n=208)$ and MMF-treated $(n=184)$ patients, 4 to 257 months after renal transplantation. Population pharmacokinetic analysis was performed using nonlinear mixed-effects modeling (NONMEM). A two-compartment model with first-order absorption and elimination was used to describe the data.

Results: No differences were detected in MPA clearance, intercompartmental clearance, central- or peripheral volume of distribution. Respective values and inter-patient variability (IPV) were; $16 \mathrm{~L} / \mathrm{hr}$ (39\%), $22 \mathrm{~L} / \mathrm{hr}$ (78\%), $40 \mathrm{~L}$ (100\%) and 518 L (494\%). EC-MPS was absorbed more slowly than MMF with respective values of $3.0 \mathrm{hr}^{-1}$ versus $4.1 \mathrm{hr}^{-1}$ ( $p<0.001$ )(IPV 136\%). A mixture model was used for the change-point parameter lag-time $\left(T_{\text {lag }}\right)$ in order to describe IPV in this parameter adequately for EC-MPS.
Following the morning dose of EC-MPS $\mathrm{T}_{\text {lag }}$ was $0.95,1.9$ and $4.8 \mathrm{hr}$ for $51 \%, 32 \%$ and $17 \%$ of the population (IPV $8 \%$ ). The morning $\mathrm{T}_{\text {lag }}$ following EC-MPS was significantly different from both the $\mathrm{T}_{\text {lag }}$ of MMF $\left(0.30 \mathrm{hr}, \mathrm{p}<0.001\right.$ (IPV 11\%)) and the $\mathrm{T}_{\text {lag }}$ following the evening dose of EC-MPS $(9.0 \mathrm{hr}, \mathrm{p}<0.001$ (IPV $40 \%)$ ). Post-hoc analysis showed that $\mathrm{T}_{\text {lag }}$ was longer and more variable after EC-MPS administration (morning: median $1.95 \mathrm{hr}$ (range 0.95-5.5 hr), evening: median $8.87 \mathrm{hr}$ (range 5.4-12.3 hr)) compared to MMF administration (median $0.30 \mathrm{hr}$ (range 0.26$0.34 \mathrm{hr}$ ), $\mathrm{p}<0.001$ ), see figure. Following EC-MPS administration MPA predose levels were higher and more variable than following MMF administration, with respective values of $2.6 \mathrm{mg} / \mathrm{L}(0.4-$ $24.4 \mathrm{mg} / \mathrm{L})$ and $1.6 \mathrm{mg} / \mathrm{L}(0.2-7.6 \mathrm{mg} / \mathrm{L})$. The correlation between predose levels and AUC was lower in EC-MPS treated patients $\left(r^{2}=0.02\right)$ than in MMF treated patients $\left(r^{2}=0.48\right)$.

Conclusion: Absorption of MPA is delayed and varies more in EC-MPS treated patients. The greater variability in the absorption profile causes a wider range in predose concentrations and a lower correlation with exposure. This may have major consequences for MPA monitoring.

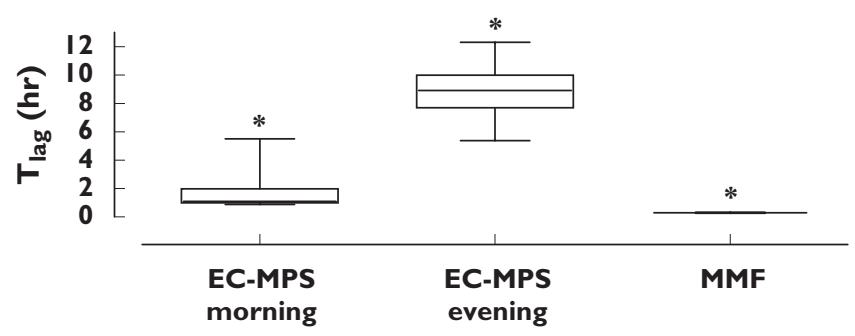

\section{Figure}

Boxplot of posthoc lag-time $\left(\mathrm{T}_{\text {lag }}\right)$. The box represents the median and the 25 and 75 percent quartiles. ${ }^{*} \mathrm{p}<0.001$.

\section{Does the number of previous vaginal deliveries affect overactive bladder symptoms or their response to treatment?}

\author{
L. P. W. Witte, ${ }^{1}$ U. Peschers, ${ }^{2}$ M. Vogel, ${ }^{3}$ J. J. M. C. H. de la Rosette' \\ \& M. C. Michel ${ }^{1}$ \\ 'Depts. of Pharmacology \& Pharmacotherapy and of Urology, \\ Academic Medical Center, University of Amsterdam, Amsterdam, The \\ Netherlands, ${ }^{2}$ Pelvic Floor Center, Munich, Germany, ${ }^{3}$ Astellas Pharma \\ GmbH, Munich, Germany
}

Introduction: Childbirth, particularly vaginal delivery is a well known risk factor for stress urinary incontinence. A relationship between pregnancies and urge urinary incontinence has also been proposed for Asian populations [Lapitan, 2001; Zhang, 2006], but little data is available for Caucasian women. The present study has used the database of a previously published, large-scale observational study into the use of solifenacin in overactive bladder syndrome (OAB) patients [Michel, 2008] to explore associations between number of vaginal deliveries and $O A B$ symptoms. As a secondary aim we have explored a possible association between deliveries and the therapeutic response to solifenacin. Finally, we also compared males and females with regard to OAB symptoms and therapeutic responses.

Methods: This study is a pre-planned secondary analysis of a published study [Michel, 2008]. 4450 Patients were evaluated who had received solifenacin treatment $(5-10 \mathrm{mg})$ for 12 weeks by 1316 office-based urologists based upon their medical judgment. Ethnicity of patients in this study was largely Caucasian. Captured variables were episode frequencies of the classical $O A B$ 
symptoms frequency, nocturia, urgency and incontinence and various validated $O A B$ rating scales.

Significance of differences was assessed by one-way analysis of variance with multiple comparison-corrected post-tests with women without a history of vaginal deliveries being the reference group; $\mathrm{a} p<0.05$ was considered significant.

Results: Women without, with 1,2 or more than 2 vaginal deliveries and men were similar in their baseline characteristics. In the overall group of patients, solifenacin treatment was associated with the following changes of OAB symptoms: the number of micturitions $/ 24 \mathrm{~h}$ decreased by $39 \%$, the number of nocturia episodes $/ 24$ by $59 \%$, the number of urgency episodes $/ 24$ by $63 \%$, and the number of incontinence episodes/24 by $73 \%$; concomitantly, pad use/ $24 \mathrm{~h}$ decreased by $59 \%$, and micturition volume increased by $67 \%$. As compared to women without vaginal deliv- eries, women with 1,2 or more deliveries and also men exhibited rather similar reductions in symptoms and improvements in rating scales upon treatment.

Conclusions: At least in a Caucasian population, gender or number of vaginal deliveries do not exhibit pathophysiologically informative differences between groups. To the best of our knowledge no previous study has assessed the potential role of parity in the treatment responses to a muscarinic receptor antagonist in $\mathrm{OAB}$ patients. Our data indicate rather similar treatment responses to the muscarinic receptor antagonist solifenacin irrespective of gender and number of vaginal deliveries.

1 Lapitan MC et al., Int Urogynecol J (2001) 12; 226-231. 2 Zhang W et al., Neurourol Urodyn (2006) 25; 717-721. 3 Michel MC et al., Drug Safety (2008) 31; 505-514.

\section{Safe and effective 6-thioguanine treatment of a thiopurine S-methyltransferase deficient Crohn's disease patient using therapeutic drug monitoring \\ D. R. Wong, ${ }^{1}$ W. G. N. Mares, ${ }^{2}$ L. P. L. Gilissen, ${ }^{3}$ A. A. M. Masclee, ${ }^{2}$ \\ L. G. J. B. Engels, ${ }^{4}$ C. Neef ${ }^{5}$ \& P. M. Hooymans ${ }^{1}$ \\ 'Clinical Pharmacy, Orbis Medical Centre, Sittard ' Gastroenterology \\ and Hepatology, University Hospital Maastricht, Maastricht, \\ ${ }^{3}$ Gastroenterology and Hepatology, Catharina Hospital, Eindhoven, \\ ${ }^{4}$ Gastroenterology and Hepatology, Orbis Medical Centre, Sittard \\ ${ }^{5}$ Clinical Pharmacy, University Hospital Maastricht, Maastricht The \\ Netherlands}

Introduction: The immunosuppressive thiopurines, azathioprine (AZA) and 6-mercaptopurine (6-MP), have proven efficacy in steroid-dependant or refractory inflammatory bowel disease (IBD). Thiopurine S-methyl-transferase (TPMT) is one of the most important enzymes in thiopurine metabolism. In case of TPMT deficiency serious myelosuppression may occur. Recently, another thiopurine drug, 6-thioguanine (6-TG), has been suggested in case of AZA -and 6-MP resistant or intolerant patients.

Case description: We report the case of a 33-year-old man with a complicated history of severe fistulizing ileocolonic Crohn's disease. He was unsuccessfully treated with sulfasalazine and prednisolone, and later mesalazine monotherapy. 6-MP was started (50 mg daily; $0.64 \mathrm{mg} / \mathrm{kg}$ body weight). Four weeks later the patient was admitted to the hospital for severe pancytopenia (hemoglobin $4.3 \mathrm{mmol} / \mathrm{l}$, thrombocytes $24 * 10^{9} / \mathrm{l}$, leukocytes $\left.0.9 * 10^{9} / \mathrm{l}\right)$. The active 6-MP metabolites 6thioguaninenucleotides (6-TGN) were myelotoxic (1282 pmol/ $8 * 10^{8} \mathrm{RBC}$; therapeutic (6-MP): $235-500 \mathrm{pmol} / 8 * 10^{8} \mathrm{RBC}$ ), whereas the hepatotoxic 6-methyl-mercaptopurine ribonucleotides (6-MMPR) were negative (therapeutic (6-MP): $<5700 \mathrm{pmol} /$ $\left.8 * 10^{8} \mathrm{RBC}\right)$.

Phenotyping revealed no TPMT-activity (genotype: TPMT *3A/*3A). 6-MP was discontinued and the patient was treated with erythrocyte and thrombocyte transfusions. Two months later blood constituents normalised. The ileocutaneous fistulae were treated with infliximab infusions ( $5 \mathrm{mg} / \mathrm{kg}$ body weight) every 8 weeks with temporary improvement. Fistulae output decreased after infliximab infusions, but increased some weeks before the next infusion. One and a half year later, regarding the TPMT deficiency, a low dose of 6-TG $(20 \mathrm{mg} /$ week, $0.036 \mathrm{mg} / \mathrm{kg}$ body weight daily) was added. 6-TGN levels and complete blood counts were monitored every week. After three weeks the 6TGN level raised to $1003 \mathrm{pmol} / 8 * 10^{8} \mathrm{RBC}$ (therapeutic (6-TG): $<1000 \mathrm{pmol} / 8^{*} 10^{8} \mathrm{RBC}$ ), so that the 6 -TG dose was lowered to $20 \mathrm{mg}$ every two weeks ( $0.018 \mathrm{mg} / \mathrm{kg}$ body weight daily). 6-TGN steady-state levels obtained were between 600 and $900 \mathrm{pmol} /$ $8 * 10^{8}$ RBC. Three months after starting 6-TG the fistulae closed. The patient is in clinical remission for 2.5 years now and complete blood counts and liver tests remained normal.

Discussion: In contrast to AZA and 6-MP, 6-TG is directly converted to the active 6-TGN, without formation of 6-MMPR by TPMT.6-TG has been reported to be a safe and effective alternative in AZA/ 6-MP intolerant - and resistant IBD patients. However, it is generally recommended that TPMT deficient IBD patients should not be treated with thiopurines.

Conclusion: Our case demonstrates that very low dose 6-TG under close clinical surveillance and frequent therapeutic drug monitoring, may be a rescue drug for IBD-patients with low or without functional TPMT activity. 